\title{
Hydroxyapatite deposition on molecular assemblies of a porphyrin derivative under body fluid conditions
}

\author{
Mineo HASHIZUME ${ }^{*, *, \dagger}$ and Ryota SAITO ${ }^{* *}$ \\ *Department of Industrial Chemistry, Faculty of Engineering, Tokyo University of Science, \\ 12-1 Ichigayafunagawara-machi, Shinjuku-ku, Tokyo 162-0826 \\ ** Graduate School of Chemical Sciences and Technology, Tokyo University of Science, \\ 12-1 Ichigayafunagawara-machi, Shinjuku-ku, Tokyo 162-0826
}

\begin{abstract}
Biomimetic mineralization is useful to deposit biominerals on various organic scaffolds under mild conditions. This study investigated the use of molecular self-assemblies as scaffolds for hydroxyapatite (HAp) deposition under body fluid conditions. We also evaluated the scaffold assembly structures during mineralization. A porphyrin derivative having carboxylic acid groups (TCPP) was adsorbed from buffer solutions onto solid substrates to form TCPP self-assemblies. The resulting substrates were then immersed in 1.5SBF, a solution having 1.5 times higher inorganic ion concentrations than those of simulated body fluid (SBF). They were then incubated at $36.5^{\circ} \mathrm{C}$. The obtained samples' surfaces were examined using various physical characterizations such as scanning electron microscopy, FT-IR spectroscopy, and thin-film X-ray diffraction. Furthermore, the samples' surfaces were monitored spectroscopically during HAp deposition, particularly addressing the Soret band peaks of TCPP assemblies that are sensitive to TCPP assembly structures. Results showed that the TCPP assemblies formed on 2-aminoethanethiol-modified solid substrates induced heterogeneous nucleation and growth HAp on their surfaces while maintaining their J-aggregate structures. No marked effect was observed for TCPP assembly structures during HAp deposition when polyelectrolyte thin films were introduced as a soft layer between the TCPP assemblies and solid substrates, which supported the structural rigidity of the TCPP assemblies against HAp formation thereon.
\end{abstract}

(2011 The Ceramic Society of Japan. All rights reserved.

Key-words : Biomineralization, Organic-inorganic hybrid, Porphyrin, Self-assembly, Hydroxyapatite, Simulated body fluid

[Received February 9, 2011 ; Accepted March 30, 2011]

\section{Introduction}

Preparation of organic-inorganic hybrids using biomimetic mineralization on organic scaffolds is useful because it proceeds under mild conditions. ${ }^{1)-3)}$ Much effort has been devoted to this field from the viewpoint of preparation of useful hybrid materials for biomedical application and that of creation of novel organicinorganic nanohybrids. For example, various states of organic materials such as solid substrates ${ }^{4)}$ and films, ${ }^{5), 6)}$ fibers, ${ }^{77,8)}$ porous materials, ${ }^{9)}$ gels, ${ }^{10)}$ colloidal particles ${ }^{11)}$ self-assembled monolayers, ${ }^{12)}$ and protein layers ${ }^{13), 14)}$ have been used as scaffolds for hybridization with hydroxyapatite (HAp) using simulated body fluid (SBF) developed by Kokubo et al. ${ }^{15), 16)}$ and $1.5 \mathrm{SBF}$, a solution having 1.5 times higher ion concentrations than those of $\mathrm{SBF}^{3)}$ Because SBF mimics body fluid conditions, it is useful to evaluate the in vivo HAp deposition ability of materials. Actually, HAp deposited from SBF is much more bioactive than that obtained from conventional solution precipitation processes because of their partial ion substitution and lower crystallinity.

In this study, we investigated HAp deposition on scaffolds consisting of supramolecular assemblies of a porphyrin derivative having carboxylic acid groups-tetrakis(4-carboxyphenyl)porphine (TCPP) - formed on solid substrates under body fluid conditions (Fig. 1). Several examples exist for creation of organic-inorganic nanohybrids consisting of supramolecular assemblies of porphyrin derivatives and inorganic components using sol-gel processes. ${ }^{17), 18)}$ However, such hybrids prepared

$\dagger$ Corresponding author: M. Hashizume; E-mail: mhashizu@ci. kagu.tus.ac.jp

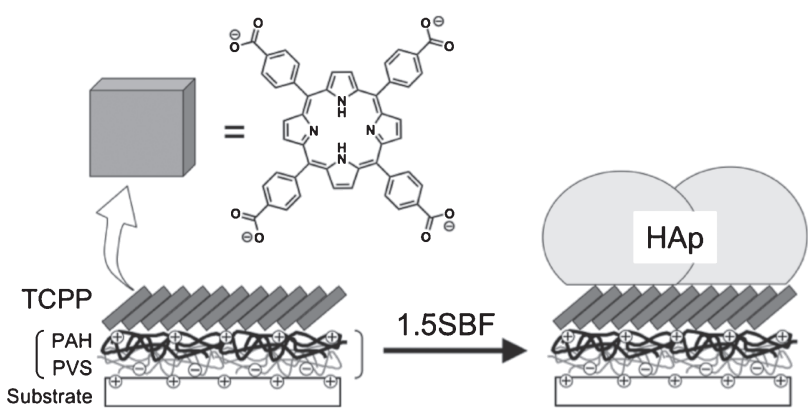

Fig. 1. Schematic illustration of HAp deposition on the molecular assembly of TCPP formed on a substrate under body fluid conditions.

using biomimetic mineralization have not been reported in the relevant literature. Porphyrin derivatives have unique spectroscopic characteristics that are sensitive to their microscopic assembly structures. ${ }^{19)}$ We evaluated the availability of supramolecular dye assemblies constructed through noncovalent interactions as scaffolds for biomimetic HAp deposition. We also specifically examined whether HAp deposition processes affect molecular assembly structures of the scaffolds.

\section{Experimental procedures}

\subsection{Chemicals}

TCPP and 3-aminopropyltriethoxysilane (APS) were obtained from Tokyo Chemical Industry Co., Ltd. 2-Aminoethanethiol hydrochloride, potassium polyvinyl sulfate (PVS, molecular weight ca. 240000) were purchased from Wako Pure Chemical Industries, Ltd. Poly(allylamine hydrochloride) (PAH, avg. $M_{\mathrm{w}}$ ca. 65000) was obtained from Sigma-Aldrich Chemical Co., Ltd. 
Other chemicals, including those for preparation of $1.5 \mathrm{SBF}$ were obtained from Nacalai Tesque Inc. All chemicals were used as received. According to Kokubo's method, 1.5SBF $\left(\mathrm{Na}^{+} 213.0\right.$, $\mathrm{K}^{+} 7.5, \mathrm{Mg}^{2+}$ 2.3, $\mathrm{Ca}^{2+} 3.8, \mathrm{Cl}^{-} 221.7, \mathrm{HCO}_{3}{ }^{-}$6.3, $\mathrm{HPO}_{4}{ }^{2-}$ 1.5, and $\mathrm{SO}_{4}{ }^{2-} 0.8 \mathrm{mM}, \mathrm{pH}$ 7.4) was prepared. ${ }^{15), 16)}$ Distilled water and ultrapure water were prepared for experiments (RFD210TA and RFU414BA, respectively; Advantec Toyo Kaisha Ltd.).

\subsection{Formation of TCPP assembly on substrates}

The TCPP solutions $\left(10^{-7}-10^{-5} \mathrm{M}\right)$ were prepared by dissolving proper amounts of TCPP in $1 \mathrm{mM}$ Tris- $\mathrm{HCl}$ buffer ( $\mathrm{pH} 7.25)$. For UV-Vis reflection absorption spectroscopy, gold-coated glass substrates modified by 2-aminoethanethiol were used. For UVVis transmission spectroscopy, quartz plates were used as the substrate. The plates were cleaned using $\mathrm{EtOH} / \mathrm{KOH}$ treatment with subsequent silane coupling using APS according to a company protocol. ${ }^{20)}$ In some cases, on the cationic surfaces of these substrates, an ultrathin layer of PVS and a PAH layer were laminated sequentially using conventional layer-by-layer assembly. ${ }^{21), 22)}$ Therefore, the resulting surfaces were also positively charged. These substrates with or without polymer laminations were immersed in the TCPP solutions to form self-assembled monolayers of TCPP. The substrates were then rinsed with ultrapure water and dried using gentle nitrogen flushing.

\subsection{HAp deposition on TCPP-adsorbed sub- strates}

The TCPP-adsorbed PS substrates with and without introduced $\mathrm{PAH} / \mathrm{PVS}$ interlayers were immersed in $1.5 \mathrm{SBF}$ at $36.5^{\circ} \mathrm{C}$ and incubated using a dry chamber (DOV-750A; AS ONE Corp.). After an appropriate time, the substrates were picked up, rinsed with ultrapure water, and dried using gentle nitrogen flushing.

\subsection{Characterization}

UV-Vis transmission spectroscopy for the samples was conducted using a spectrophotometer (U-2000A; Hitachi Ltd.). The UV-Vis reflection absorption spectra of the samples were recorded using a spectrophotometer (incident angle $30^{\circ}$, V-570; JEOL). The absorbance was recorded as the relative reflectivity $\left[\% R=R_{\infty}(\right.$ sample $) / R_{\infty}$ (reference) $]$. The FT-IR spectra of the samples were recorded using a single-reflection attenuation totalreflection (ATR) apparatus (Nicolet 380 combined with OMNIsampler module; Thermo Fisher Scientific Inc.). The surface morphology and elemental composition of the resulting samples were evaluated using a scanning electron microscopy (SEM, S-5000; Hitachi Ltd.) and energy-dispersive X-ray spectroscopy (EDX, Sigma; Kevex) with acceleration voltage of $10 \mathrm{kV}$. In some cases, the specimens were sputtered with Pt-Pd using an ion sputter (E-1030; Hitachi Ltd.) to prevent charge-up. Thin-film $\mathrm{X}$-ray diffraction (TF-XRD) studies were conducted $(\mathrm{CuK} \alpha$, $40 \mathrm{kV}, 40 \mathrm{mV}$, step $0.02^{\circ}$, RINT-2200VL; Rigaku Corp.).

\section{Results and discussion}

\subsection{Preparation and characterization of TCPP monolayer assemblies on solid substrates}

Porphyrin derivatives, including TCPP, have characteristic absorption maxima at around $400 \mathrm{~nm}$ (Soret band) caused by their expanded $\pi$-systems, which are sensitive to their aggregation structures. ${ }^{19)}$ The transmission spectra of TCPP in a buffer solution showed the peak of the Soret band at $414 \mathrm{~nm}$ [Fig. 2(a), denoted by asterisk], which confirmed that TCPP was molecularly dissolved in the solution. However, the Soret band peaks appeared at $442 \mathrm{~nm}$ in the reflection absorption spectra of TCPP assemblies

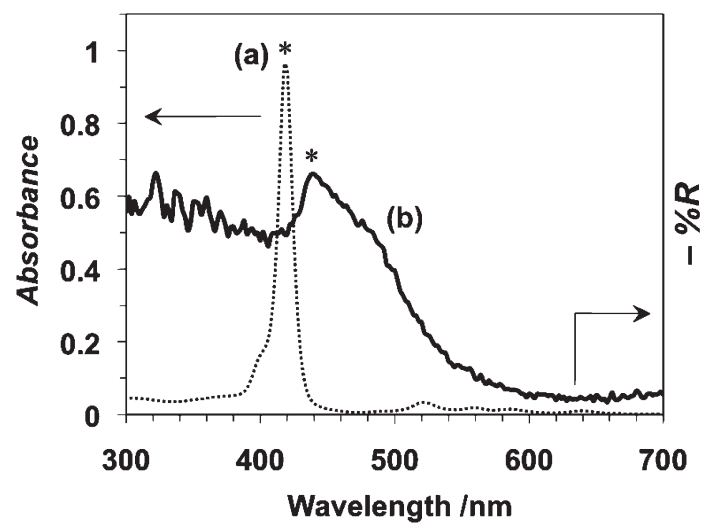

Fig. 2. (a) UV-Vis transmission spectra of TCPP $\left(2 \times 10^{-6} \mathrm{M}\right)$ in Tris$\mathrm{HCl}$ buffer ( $\mathrm{pH}$ 7.25) (scale: left axis). (b) UV-Vis reflection absorption spectra of TCPP assemblies formed on 2-aminoethanethiol-modified substrate (scale: right axis). In both (a) and (b), Soret band peaks are denoted by asterisk $\left(^{*}\right)$.

Table 1. Wavelength of Soret band absorption maxima for different states of TCPP

\begin{tabular}{lc}
\hline \multicolumn{1}{c}{ States of TCPP } & $\begin{array}{c}\text { Peak at } \\
\text { Soret band } \\
\text { /nm }\end{array}$ \\
\hline In Tris-HCl buffer $(\mathrm{pH} 7.25)\left([\mathrm{TCPP}]=2 \times 10^{-6} \mathrm{M}\right)$ & 414 \\
On 2-aminoethanethiol-modified gold substrate & 442 \\
On PAH/PVS/2-aminoethanethiol-modified gold substrate & 436 \\
On APS-modified glass substrate & 422 \\
On PAH/PVS/APS-modified glass substrate & 425 \\
\hline
\end{tabular}

on 2-aminoethanethiol-modified gold substrates [Fig. 2(b), denoted by asterisk]. These red-shifts of Soret bands supported TCPP formation of J-aggregates on these substrate surfaces. ${ }^{19)}$ The transmission spectra for TCPP assemblies on APS-modified quartz substrates also exhibited a red-shift of the Soret bands (data not shown). The absorption maxima of the Soret bands for TCPP in the solution and on the substrates are presented in Table 1, which clearly shows that TCPP formed J-aggregates on these solid substrates. No effect of introducing the PAH/PVS interlayer between TCPP assemblies and 2-aminoethanethiol-modified gold substrates or APS-modified quartz substrates on the assembling structure of TCPP is apparent. Although differences were found in the degree of red-shift between TCPP assemblies on quartz substrates and those on gold substrates, this probably arose from the difference in measurement methods (transmission spectra and reflection spectra) because both quartz and gold substrates had positively charged surfaces by molecular layer-forming surface modification agents (2-aminoethanethiol and APS). Considered from the molecular shape of TCPP and the results of UV-Vis spectroscopy, it was inferred that TCPP formed monolayer assemblies on solid substrates as portrayed in Fig. 1. Such assembly states on oppositely charged substrates were also observed in the cases of other porphyrin derivatives having ionic functional groups at their meso-positions. ${ }^{22)}$

\subsection{HAp deposition on TCPP-adsorbed sub- strates in 1.5SBF}

The TCPP assembly-adsorbed 2-aminoethanethiol-modified gold substrates were then immersed in $1.5 \mathrm{SBF}$ and incubated to evaluate their HAp deposition abilities. SEM images of the resulting samples revealed that hemispherical deposits were formed on their surfaces [Fig. 3(a)]. Similar deposits were also 


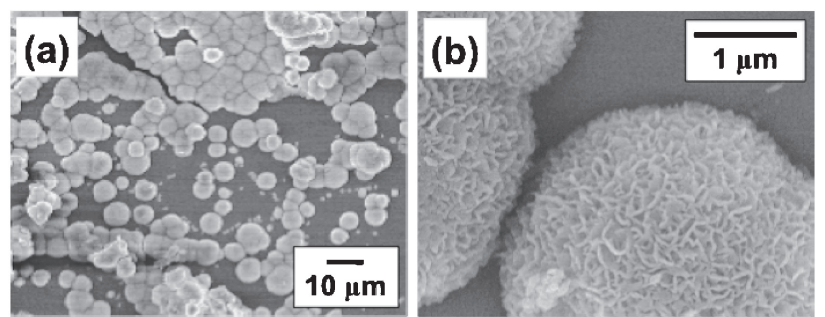

Fig. 3. SEM images of TCPP assembly-adsorbed 2-aminoethanethiolmodified substrate (a) and TCPP assembly-adsorbed PAH/PVS/2-aminoethanethiol-modified substrate (b) after immersion in $1.5 \mathrm{SBF}$ for 7 days.

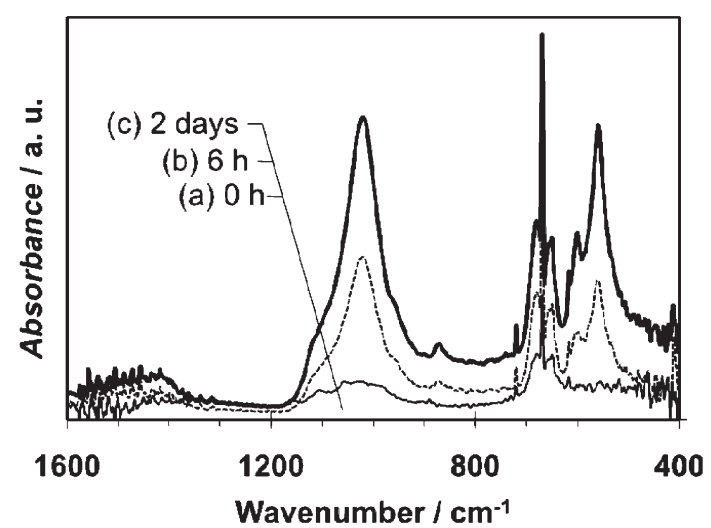

Fig. 4. FT-IR spectra of TCPP assembly-adsorbed 2-aminoethanethiolmodified substrates: (a), before immersion in 1.5SBF ( $0 \mathrm{~h}$, solid line); (b) after immersion in $1.5 \mathrm{SBF}$ for $6 \mathrm{~h}$ (dashed line); and (c) after immersion in $1.5 \mathrm{SBF}$ for 2 days (bold solid line).

observed for the case of TCPP-assemblies adsorbed onto PAH/ PVS/2-aminoethanethiol-modified substrates. For both systems, it is difficult to evaluate the difference in ability to induce formation of such depositions in 1.5SBF under current experimental conditions. Magnified SEM images of the deposits showed that they consisted of plate-like objects, which were characteristic morphologies to HAp deposited from SBFs [Fig. 3(b)]. ${ }^{3-16)}$ The EDX spectra of the corresponding SEM images showed that these deposits contained calcium and phosphorus. The $\mathrm{Ca} / \mathrm{P}$ ratios for these samples were not accurately obtainable because the peak of phosphorus was largely overlapped with that of gold originated from the substrate (data not shown). The FT-IR spectra of the surfaces of TCPPassemblies adsorbed onto 2-aminoethanethiol-modified substrates exhibited two intense absorption peaks in the region of about $1150-900 \mathrm{~cm}^{-1}$ and $650-500 \mathrm{~cm}^{-1}$, which were respectively assignable to the stretching vibration $\left(v_{3}\right)$ of the phosphate $\left(\mathrm{PO}_{4}{ }^{3-}\right)$ groups and the bending vibration $\left(v_{4}\right)$ of the phosphate $\left(\mathrm{PO}_{4}{ }^{3-}\right)$ groups (Fig. 4). ${ }^{23)-25)}$ The intensities of these peaks increased concomitantly with increased incubation time. The shapes of these peaks were broader than those of stoichiometric HAp having high crystallinity. ${ }^{23)-25)}$ A slight increase of the broad absorption peak at $1550-1400 \mathrm{~cm}^{-1}$ after 2 days' incubation indicated that the deposits contained $\left.\left.\mathrm{CO}_{3}{ }^{2-} \cdot 26\right), 27\right)$ For these samples, higher wavenumber regions $\left(4000-3500 \mathrm{~cm}^{-1}\right)$, where the $\mathrm{OH}$ stretching vibration (about $3570 \mathrm{~cm}^{-1}$ ) characteristic to $\mathrm{HAp}^{26)}$ might appear, were difficult to evaluate in detail because of the low resolution of the present measurement method (singlereflection ATR). The TH-XRD patterns for the case of TCPPassemblies adsorbed onto 2-aminoethanethiol-modified substrates showed two peaks at $2 \theta=26$ and $32^{\circ}$, which were respectively assigned to the 002 diffraction line of HAp and an

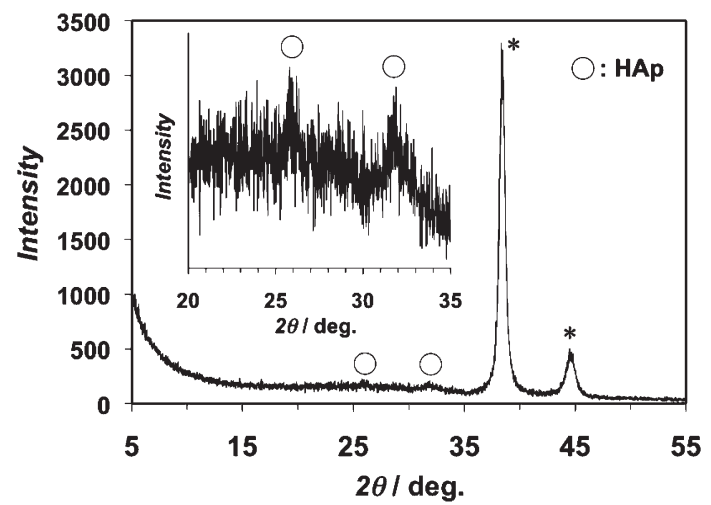

Fig. 5. XRD pattern of TCPP assembly-adsorbed 2-aminoethanethiolmodified substrate after immersion in $1.5 \mathrm{SBF}$ for 7 days and its magnification (inset). Peaks marked with an open circle correspond to those of HAp (see the text). Other peaks respectively appeared at $2 \theta=38$ and $44.5^{\circ}$ [denoted by asterisk $(*)$ ] originate from 111 and 200 diffraction of gold (PDF\#04-0784) ${ }^{28)}$ in the substrate.

envelope of the 211, 112, and 300 diffraction line of HAp (PDF\#09-0432) (Fig. 5). ${ }^{28)}$ These results strongly support that TCPP assemblies formed on positively charged solid substrates induced heterogeneous nucleation and growth of HAp in 1.5SBF, which is reasonable because TCPP assembly surfaces showed carboxylic acid groups arranged densely toward the solution phase (1.5SBF) when they formed monolayers, as presented in Fig. 1. The shapes of peaks assignable to $\mathrm{PO}_{4}{ }^{3-}$ and the appearance of the peak in the region of about $1550-1400 \mathrm{~cm}^{-1}$ in the FT-IR spectra, and intensities of the diffraction peaks in the TH-XRD patterns indicate that the obtained HAp deposits were bone-like: that is, the HAp was partly carbonated and had low crystallinity. Under current conditions, no marked difference was observed for HAp deposition behaviors between the samples with and without the PAH/PVS interlayers.

\subsection{Effect of HAp deposition on assembling struc- tures of TCPP}

The effects of HAp nucleation and growth processes on microscopic structures of TCPP assemblies were evaluated spectroscopically because formation of rigid inorganic crystals may affect interfacial structures of scaffolds, especially when they are constructed through noncovalent interactions (molecular self-assembly). Figure 6(a) shows UV-Vis reflection absorption spectra of the TCPP assemblies on 2-aminoethanethiol-modified substrates before and after immersion in 1.5SBF. In fact, UV-Vis transmission spectroscopy yielded no useful information because of the much larger scattering effect by deposited HAp (data not shown). However, we were able to detect the Soret band peaks, which were not shifted from those before incubation, for samples after $6 \mathrm{~h}$ incubation [Fig. 6(a), solid line and dashed line]. These results support that TCPP assemblies maintained their J-aggregate structures during biomimetic HAp deposition processes in 1.5SBF. Even for reflection spectra, the Soret band peaks were overlapped by the scattering coming from deposited HAp. Such effects became greater concomitantly with the increase of incubation time [Fig. 6(a), bold solid line (2 days' incubation)]. We regard $6 \mathrm{~h}$ incubation as sufficient to observe microscopic interfacial effects of HAp deposition on TCPP assemblies. FT-IR measurements (Fig. 4) support that a certain amount of HAp had already deposited after $6 \mathrm{~h}$ incubation.

The reason for introducing the PAH/PVS interlayers between TCPP assemblies and 2-aminoethanethiol-modified gold sub- 


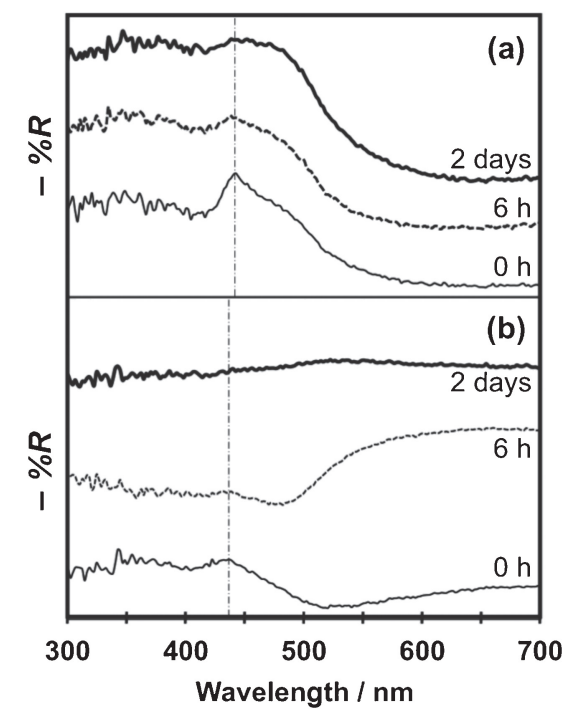

Fig. 6. UV-Vis reflection absorption spectra of TCPP assemblyadsorbed 2-aminoethanethiol-modified substrates (a) and TCPP assembly-adsorbed PAH/PVS/2-aminoethanethiol-modified substrates (b). Solid lines show data before immersed in 1.5SBF $(0 \mathrm{~h})$; dashed lines show data after immersion in $1.5 \mathrm{SBF}$ for $6 \mathrm{~h}$; bold solid lines show data after immersion in 1.5SBF for 2 days. In both (a) and (b), dashed-dotted lines show the position of the absorption maxima of the Soret band before immersion in $1.5 \mathrm{SBF}$.

strates was to add softness to the supported phase for TCPP assemblies. Because the Soret band peaks did not shift, the results imply that the TCPP assemblies that had formed on such softer surfaces maintained their J-aggregate structures during HAp deposition in 1.5SBF [Fig. 6(b)], although the Soret band peak appeared as a much smaller peak because of the large scattering effect deriving from the deposited HAp. These results support the structural rigidity of the TCPP assemblies against HAp deposition thereon. Another possibility is that the PAH/ PVS layers used in this study are too thin to act as soft layers. We are currently continuing investigations to reveal effects of the biomimetic HAp deposition process on microstructure of TCPP assemblies formed on much softer surfaces. In Fig. 6(b), the peak was almost hidden by large scattering after 2 days' incubation [Fig. 6(b), bold solid line]. It was difficult to control such scattering effects because microstructures of these surfaces affected by the deposited HAp, of which the sizes and distributions on the surfaces differed at the microscopic level. Even so, at the early stage of deposition, UV-Vis reflection absorption spectroscopy provided information for TCPP assembling structures during HAp mineralization.

\section{Conclusions}

The results obtained from this study show that TCPP assemblies formed on 2-aminoethanethiol-modified solid substrates induced heterogeneous nucleation and growth of HAp on their surfaces while maintaining their J-aggregate structures when incubated in 1.5SBF. Introduction of ultrathin PAH/PVS layers between the TCPP monolayers and 2-aminoethanethiolmodified substrate did not affect the TCPP assembly microstructures. They acted as rigid scaffolds for HAp deposition. This study demonstrates the potential use of molecular self-assemblies as scaffolds for biomimetic HAp deposition.

Acknowledgements The authors gratefully acknowledge Prof. T. Kawai (Tokyo University of Science) for preparation of gold- coated glass substrates and for UV-Vis reflection absorption spectroscopy, and Dr. M. Kamitakahara (Tohoku University) for TF-XRD measurements. This work was partly supported by Research Funds awarded by the President of Tokyo University of Science (Research Fund of Tokyo University of Science, 2009).

\section{References}

1) L. A. Estroff, Ed., "Thematic Issue: Biomineralization", Chem. Rev., 108, 4329-4978 (2008).

2) E. Ruiz-Hitzky, K. Ariga and Y. Lvov, Bio-inorganic hybrid nanomaterials, Weinheim, Wiley-VCH (2008).

3) C. Ohtsuki, M. Kamitakahara and T. Miyazaki, J. Tissue Eng. Regen. Med., 1, 33-38 (2007).

4) M. Tanahashi, T. Yao, T. Kokubo, M. Minoda, T. Miyamoto, T. Nakamura and T. Yamamuro, J. Am. Ceram. Soc., 77, 28052808 (1994).

5) W. L. Murphy and D. J. Mooney, J. Am. Chem. Soc., 124, 1910-1917 (2002).

6) T. Kawai, C. Ohtsuki, M. Kamitakahara, T. Miyazaki, M. Tanihara, Y. Sakaguchi and S. Konagaya, Biomaterials, 25, 4529-4534 (2004).

7) A. Oyane, M. Kawashita, K. Nakanishi, T. Kokubo, M. Minoda, T. Miyamoto and T. Nakamura, Biomaterials, 24, 1729-1735 (2003).

8) J. Chen, B. Chu and B. S. Hsiao, J. Biomed. Mater. Res., 79A, 307-317 (2006).

9) W. L. Murphy, D. H. Kohn and D. J. Mooney, J. Biomed. Mater. Res., 50, 50-58 (2000).

10) K. Hosoya, C. Ohtsuki, T. Kawai, M. Kamitakahara, S. Ogata, T. Miyazaki and M. Tanihara, J. Biomed. Mater. Res., 71A, 596-601 (2004).

11) M. Hashizume, H. Horii, J. Kikuchi, M. Kamitakahara, C. Ohtsuki and M. Tanihara, J. Mater. Sci.: Mater. Med., 21, 11-19 (2010).

12) M. Tanahashi and T. Matsuda, J. Biomed. Mater. Res., 34, 305315 (1997).

13) M. Hashizume, A. Sakai, Y. Sakamoto, H. Matsuno and T. Serizawa, Chem. Lett., 39, 220-222 (2010).

14) M. Hashizume, Y. Sakamoto, A. Sakai, H. Matsuno and T. Serizawa, Trans. Mater. Res. Soc. Jpn., 35, 115-118 (2010).

15) T. Kokubo, H. Kushitani, S. Sakka, T. Kitsugi and T. Yamamuro, J. Biomed. Mater. Res., 24, 721-734 (1990).

16) T. Kokubo and H. Takadama, Biomaterials, 27, 2907-2915 (2006).

17) T. Kishida, N. Fujita, K. Sada and S. Shinkai, J. Am. Chem. Soc., 127, 7298-7299 (2005).

18) S. Nasu and K. Kuroda, J. Colloid Interface Sci., 348, 446-451 (2010).

19) R. F. Khairutdinov and N. Serpone, J. Phys. Chem. B, 103, 761-769 (1999).

20) Applying a silane coupling agent, Technical Library of Gelest, Inc., http://www.gelest.com.

21) G. Decher, Science, 277, 1232-1237 (1997).

22) K. Ariga, Y. Lvov and T. Kunitake, J. Am. Chem. Soc., 119, 2224-2231 (1997).

23) C. B. Baddiel and E. E. Berry, Spectrochim. Acta $[A]$, 22, 1407-1416 (1966).

24) C. Rey, M. Shimizu, B. Collins and M. J. Glimcher, Calcif. Tissue Int., 46, 384-394 (1990).

25) C. Rey, M. Shimizu, B. Collins and M. J. Glimcher, Calcif. Tissue Int., 49, 383-388 (1991).

26) B. Cengiz, Y. Gokce, N. Yildiz, Z. Atkas and A. Calimli, Colloids Surf., A, 322, 29-33 (2008).

27) L. Li, G. Li, Y. Wang and J. Jiang, Appl. Surf. Sci., 255, 77347738 (2008).

28) The International Centre for Diffraction Data, http://www. icdd.com. 